\title{
Laser flash photolysis study on 9-phenylxanthenium tetrafluoroborate: Identification of new features due to the triplet state
}

\author{
SANGHAMITRA BANERJEE and ANUNAY SAMANTA* \\ School of Chemistry, University of Hyderabad, Hyderabad 500 046, India \\ e-mail: assc@uohyd.ernet.in
}

MS received 8 October 2010; accepted 25 November 2010

\begin{abstract}
Laser flash photolysis study on highly fluorescent and stable salt of 9-phenylxanthenium cation in neutral condition has been carried out for the first time. A new transient absorption band of this extensively studied system that perhaps remained buried under the fluorescence envelope and hitherto undetected has been identified and attributed to the triplet state of the system. This oxygen-insensitive triplet-triplet absorption band in the 480-600 $\mathrm{nm}$ range is expected to trigger new studies exploring the reactivity of the triplet state of this system, which has so far received very little attention.
\end{abstract}

Keywords. Carbocation; fluorescence; triplet-triplet absorption; laser flash photolysis.

\section{Introduction}

The carbocation intermediates are of considerable interest in physical and organic chemistry. ${ }^{1-6}$ 9-Phenylxanthenium cation $(\mathbf{P X}+)$ is a highly fluorescent and fairly stable species, whose photophysical properties have received noticeable attention. ${ }^{7-13} \mathbf{P X}+$ can be generated from 9-phenylxanthenol (PX) in acidic environment $\left(\mathbf{p K}_{\mathbf{R}+}=1.0 \pm 0.5\right.$ for $\mathbf{P X}$ in water) (scheme 1$)^{8,14,15}$ or by photoexcitation of $\mathbf{P X}$ in polar (hydroxylic) solvent, where it undergoes adiabatic dehydroxylation in the excited-state surface. ${ }^{9,10,15}$ Wan et al. have shown that in the $\mathrm{pH}$ range of 3-12 water acts as a catalyst in this photo-induced reaction and the quantum yield of formation of $\mathbf{P X}+$ is 0.4 in 1:1 acetonitrile/water. ${ }^{8,15}$ However, the catalytic activity of water was not quantitative due to efficient quenching of the singlet excited states of both $\mathbf{P X}$ and $\mathbf{P X}+$ by water and the ground state interaction between PX and water. PX+ can also be obtained as a stable species in other acidic environment such as in nafion films and zeolite matrices. ${ }^{10}$ The driving force of adiabatic dehydroxylation of $\mathbf{P X}$ is its enhanced basicity in the excited state and strong conjugation of the oxygen lone pair to the aromatic system. Although $\mathbf{P X}+$ is less reactive in the ground state, considerable

\footnotetext{
*For correspondence
}

increase of its electrophilicity is observed in the singlet excited state. ${ }^{5}$ The reactivity of $\mathbf{P X}+$ in the first excited singlet state toward a series of alcohols, amines and ethers has been measured by monitoring the strong fluorescence of the system. ${ }^{11,16}$ The fluorescence quenching rate constants have been found to lie between $10^{7}$ and $10^{10} \mathrm{M}^{-1} \mathrm{~s}^{-1}$. 5,11,16 $\mathrm{As}$ an electron deficient system, photoexcited $\mathbf{P X}+$ undergoes electron transfer reaction with a wide variety of aromatic donor molecules producing 9-phenylxanthenyl radical. ${ }^{10,13}$ The rate constants of photo-induced electron transfer reactions, which have also been measured by monitoring the quenching of fluorescence, are close to the diffusion limited values. However, the yield of net electron transfer $\left(\phi_{e t}\right)$ process is found to be very small $(\leq 0 \cdot 02)$ due to efficient back electron transfer reaction between the products of the photo-induced electron transfer reactions, i.e., 9-phenylxanthenyl radical and radical cation of the corresponding reactant. Even though the singlet excited state of $\mathbf{P X}+$ has been studied extensively by monitoring the fluorescence of the system, barring a couple of papers by Johnston and Wong, ${ }^{17,18}$ there is hardly any literature dealing with the triplet state of this system. In these papers, the authors have characterized the triplet-triplet absorption spectra of $\mathbf{P X}+$ by generating this species from PX in the presence of varied concentrations $(1-10 \mathrm{mM})$ of trifluoroacetic acid. ${ }^{17,18}$ They found that 


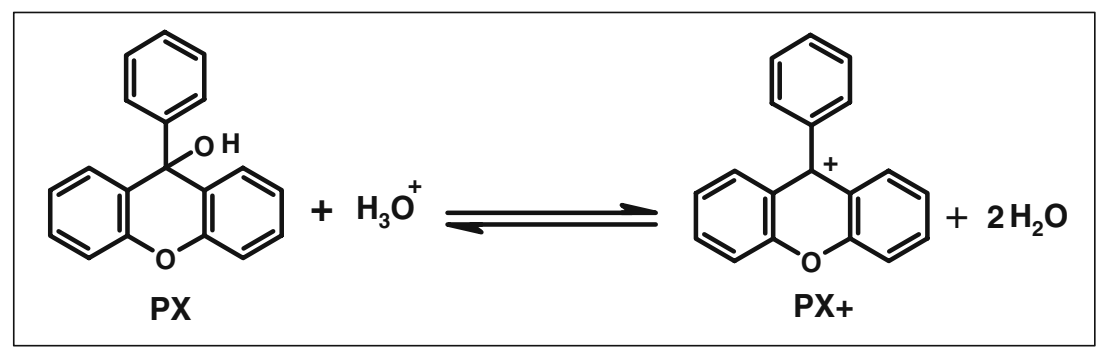

Scheme 1. Equilibrium between 9-phenylxanthenol and 9-phenylxanthenium cation.

the triplet-triplet absorption due to $\mathbf{P X}+$ shows maximum xat $<300 \mathrm{~nm}$ and is characterized by a lifetime of several microseconds. The exact lifetime depends on the concentration of $\mathbf{P X}$ in the medium (which can be varied by adjusting the concentration of the added acid) as $\mathbf{P X}+$ triplet undergoes electron transfer reaction with $\mathbf{P X}$ and produces 9-phenylxanthenyl radical, which can be characterized by the $340 \mathrm{~nm}$ band in the transient absorption spectrum. ${ }^{17,18}$ This triplet state of $\mathbf{P X}+$ can also undergo electron transfer reaction with several arene molecules. ${ }^{18}$ The lack of studies involving the triplet state of $\mathbf{P X}+$ can partly be attributed to strong fluorescence quantum yield of the species, which is reported to be 0.8 in 2,2,2-trifluoroethanol, ${ }^{18}$ a value much higher than 0.42 reported by Minto and Das (in acetonitrile containing $\left.8 \% \mathrm{H}_{2} \mathrm{SO}_{4}\right){ }^{8}$ A high fluorescence quantum yield of the system not only implies a low yield of the triplet, but the fact that the intense and broad fluorescence covers a large portion of the visible region makes observation of transient absorption due to triplet, if any, in this region a difficult exercise indeed. Minto and Das further pointed out that in addition to high fluorescence yield of $\mathbf{P X}+$, rapid electron transfer between the parent alcohol and singlet excited state of $\mathbf{P X}+$ is responsible for the absence of triplet $\mathbf{P X}+{ }^{8}$ Here, we attempt to probe the triplet state of $\mathbf{P X}+$ by following a different strategy. Unlike the earlier studies, which were performed on in situ generated PX+ (from PX) in acidic environments, we have investigated here the triplet excited state of $\mathbf{P X}+$ in non-aqueous medium and in neutral condition by preparing a stable borate salt of the cation.

\section{Experimental}

\subsection{Materials}

PX was recrystallised twice from cyclohexane before use. The tetrafluoroborate salt of $\mathbf{P X}+$ was synthesized following a standard procedure described elsewhere. ${ }^{11,19}$ In brief, PX was dissolved in propionic anhydride at $50^{\circ} \mathrm{C}$ and tetrafluoroboric acid (48 wt. \% solution in water, obtained from Aldrich) was added to it to yield bright yellow 9-phenylxanthenium tetrafluoroborate, which was washed several times by anhydrous diethylether. The solvents used in this work were of spectroscopic grade and dried by standard procedures.

9-Phenylxanthenium tetrafluoroborate: ${ }^{1} \mathrm{H}$ NMR data $\left(\mathrm{CDCl}_{3}\right): \delta 7 \cdot 75-7 \cdot 95(\mathrm{~m}, 7 \mathrm{H}), 8 \cdot 15(d, 2 \mathrm{H})$, $8 \cdot 4-8.54(\mathrm{~m}, 4 \mathrm{H}) ;{ }^{13} \mathrm{C}$ NMR: $\delta 155 \cdot 5,143 \cdot 7,132.5$, $131 \cdot 7,130 \cdot 5,130 \cdot 2,129 \cdot 1,124 \cdot 1,123 \cdot 5,120 \cdot 2,116 \cdot 5$. MP: $227^{\circ} \mathrm{C}$.

\subsection{Instrumentation}

Steady state absorption and fluorescence spectra were measured by using Cary $100 \mathrm{UV}-\mathrm{Vis}$ spectrophotometer (Varian) and Fluorolog-3 spectrofluorimeter (Horiba Jobin Yvon), respectively. Fluorescence lifetime measurements were carried out using a time correlated single photon counting (TCSPC) spectrometer (Horiba Jobin Yvon IBH), employing a laser diode $\left(\lambda_{\text {exc }}=374 \mathrm{~nm}, \mathrm{FWHM}=130 \mathrm{ps}\right)$ as the excitation source and an MCP Photomultiplier (Hamamatsu R3809U-50) as the detector.

The transient absorption measurements were performed using a laser flash photolysis set-up, which was equipped with a Q-switched Nd-YAG laser (pulse width $\sim 8 \mathrm{~ns}$ ) from Spectra Physics (Quanta-Ray INDI series) and Applied Photophysics (Model LKS60) spectrometer. The spectrometer consisted of a $150 \mathrm{~W}$ pulsed xenon lamp, programmable $\mathrm{f} / 3.4$ grating monochromator, digitized oscilloscope (Agilent, $600 \mathrm{MHz}$ ) and R-928 photomultiplier tube. The details of the setup can be found elsewhere. ${ }^{20,21}$ The solutions were excited by the third-harmonic $(355 \mathrm{~nm})$ of the laser. A perpendicular configuration was chosen for the excitation of the sample. Quartz cuvettes having a 
path length of $0.3 \mathrm{~cm}$ and $1 \mathrm{~cm}$ were used for flash photolysis studies. The freshly prepared sample solutions with optical densities kept around 0.5 at the excitation wavelength $(355 \mathrm{~nm})$ were deoxygenated by purging argon gas for about $30 \mathrm{~min}$. Applied Photophysics LKS60 Kinetic spectrometer software was used for the collection and analysis of the data.

\section{Result and discussion}

\subsection{Absorption and emission behaviour}

The absorption spectrum of yellow coloured acetonitrile solution of 9-phenylxanthenium tetrafluoroborate is shown in figure 1 . The spectrum consists of a broad band with maximum at $\sim 445 \mathrm{~nm}\left(\varepsilon_{\max } / 10^{3} \mathrm{M}^{-1} \mathrm{~cm}^{-1}=\right.$ $4 \cdot 7$ ) and relatively sharp and intense peaks at $\sim 372 \mathrm{~nm}$ $(31.9)$ and $259 \mathrm{~nm}(44 \cdot 4)$. These spectral features are consistent with the literature data. ${ }^{7-11,14,22}$ The emission spectrum of the system is characterized by a broad structureless band between 470 and $700 \mathrm{~nm}$, with maximum around $540 \mathrm{~nm}$. This emission behaviour is also similar to that of an acetonitrile solution of $\mathbf{P X}$ acidified with $8-10 \% \mathrm{H}_{2} \mathrm{SO}_{4}$ or with $6.5 \mathrm{M}$ TFA, as reported earlier. ${ }^{7-11,14,22}$ The measurement of the emission decay profile and its analysis reveal that in acetonitrile the salt exhibits a single exponential decay with lifetime of $29 \mathrm{~ns}$, a value which is comparable to the literature value of $\mathbf{P X}+{ }^{7,8,10}$

\subsection{Transient behaviour}

The transient absorption spectra of 9-phenylxanthenium tetrafluoroborate at early times (figure 2) consist

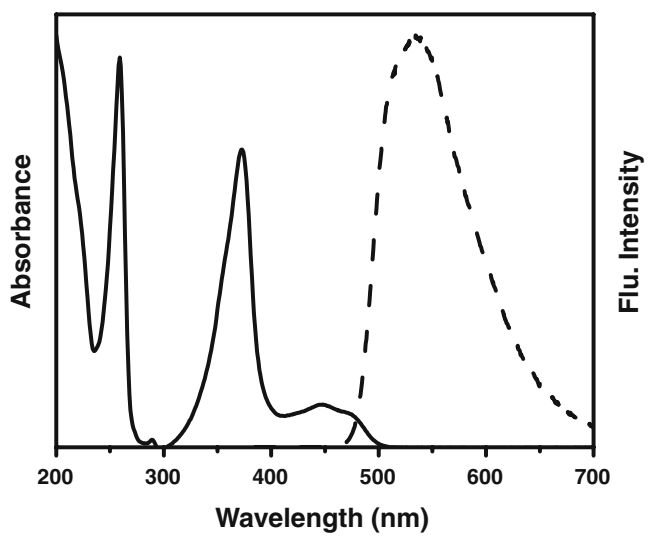

Figure 1. UV-vis absorption (solid line) and steady state fluorescence spectra (dashed line) of 9-phenylxanthenium tetrafluoroborate in acetonitrile (not scaled). $\lambda_{\text {exc }} 450 \mathrm{~nm}$, for fluorescence measurements.

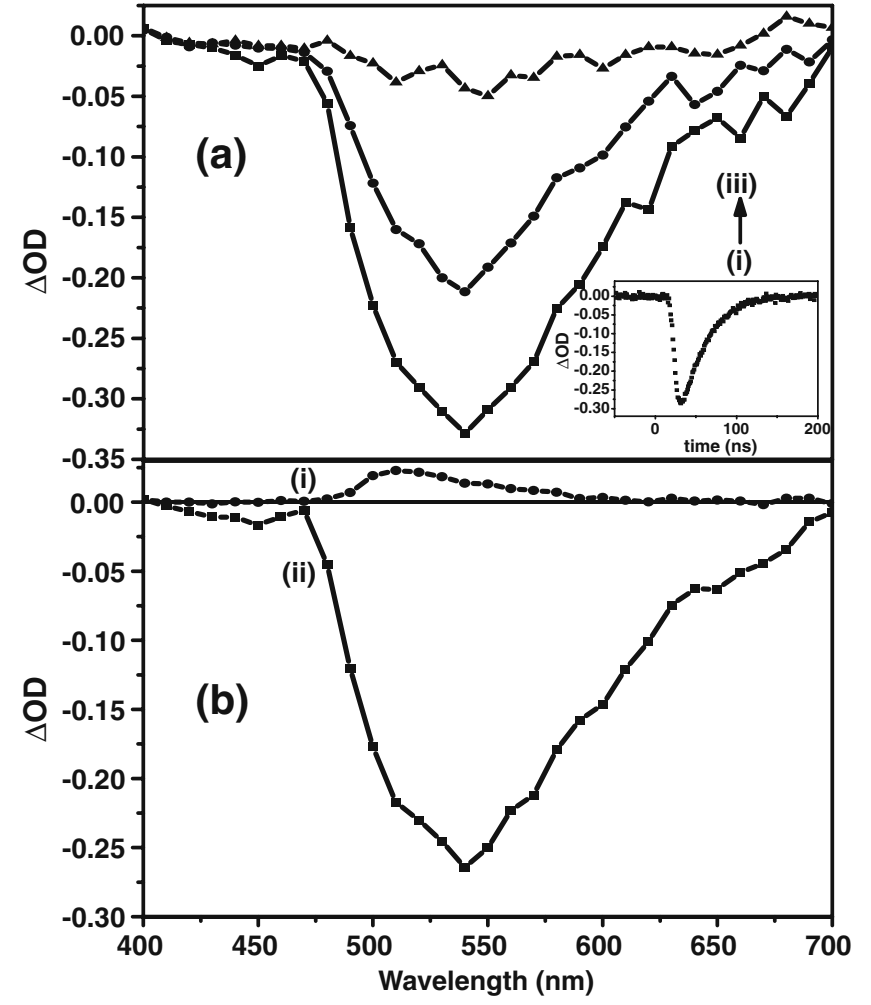

Figure 2. (a) Transient absorption spectra of 9phenylxanthenium tetrafluoroborate in acetonitrile, recorded at $\mathrm{t}=0$, (i) $20 \mu \mathrm{s}$, (ii) $60 \mu \mathrm{s}$, (iii) after $355 \mathrm{~nm}$ laser pulse excitation. The inset shows the decay profile of the $530 \mathrm{~nm}$ emission band. (b) Transient absorption spectra at $8 \mathrm{~ns}$ (i) and at $100 \mathrm{~ns}$ (ii) following the $355 \mathrm{~nm}$ laser pulse excitation of 9-phenylxanthenium tetrafluoroborate in acetonitrile.

primarily of a negative signal in the wavelength range of $400-700 \mathrm{~nm}$. An inspection of the steady state absorption and emission spectra of $\mathbf{P X}+$ suggests that strong negative signal in the $470-700 \mathrm{~nm}$ range is due to its fluorescence and relatively small negative signal between 400 and $470 \mathrm{~nm}$ is due to the ground state bleaching. The decay profile measured at $530 \mathrm{~nm}$, and is shown as an insert to figure 2(a), yielded a lifetime of $29 \mathrm{~ns}$, which is the same as the fluorescence lifetime obtained from the single photon counting measurements. ${ }^{8,10}$ Interestingly, when the same wavelength region is probed at a later time (when the fluorescence has decayed completely), one can observe an absorption band $(480-600 \mathrm{~nm})$ which is centered around $510 \mathrm{~nm}$ (figure $2 \mathrm{~b}$ ). We were really surprised how a strongly absorbing species like this one could be missed by others, particularly when taken into consideration the large number of investigations made on $\mathbf{P X}+.^{7-13,15,23}$ We carried out several control experiments to establish that this transient species is indeed a real one and not due 
to any impurities in the salt or in the solvents used in the experiments. Moreover, we generated $\mathbf{P X}+$ from an acetonitrile solution of $\mathbf{P X}$ by adding trifluoroacetic acid (the way earlier studies were conducted) and confirmed that the transient absorption behaviour of the resulting solution is also very similar to that observed with the salt solution. ${ }^{7,9-11,13,23}$ We soon figured out that this band was probably overlooked or missed due to the strong fluorescence emission that dominates in this region, particularly in the early time scale. This transient, as depicted in figure 3, survives for a reasonably long time. The lifetime of the species is estimated to be $\sim 2 \cdot 1 \mu$ s under the experimental conditions.

\subsection{Nature of the transient species}

This transient species responsible for this new absorption band can be 9-phenylxanthenyl radical, triplet state of $\mathbf{P X}$ or triplet state of $\mathbf{P X}+$. The first two possibilities can be ruled out based on literature. Minto and Das reported that the 9-phenylxanthenyl radical absorbs around $345 \mathrm{~nm}$ and is a fairly long-lived species $(\sim 150 \mu \mathrm{s}$ in air saturated $n$-heptane $)$ and the triplet of PX exhibits an absorption maximum at $\sim 440 \mathrm{~nm}$ with a lifetime of $\tau_{\mathrm{T}} \leq 0 \cdot 3 \mu$ s in acetonitrile. ${ }^{8}$ As the new transient absorption is neither due to 9-phenylxanthenyl radical nor is it due to the triplet of PX, it must be attributed to the triplet of $\mathbf{P X}+$. This assignment is confirmed by expanding the wavelength

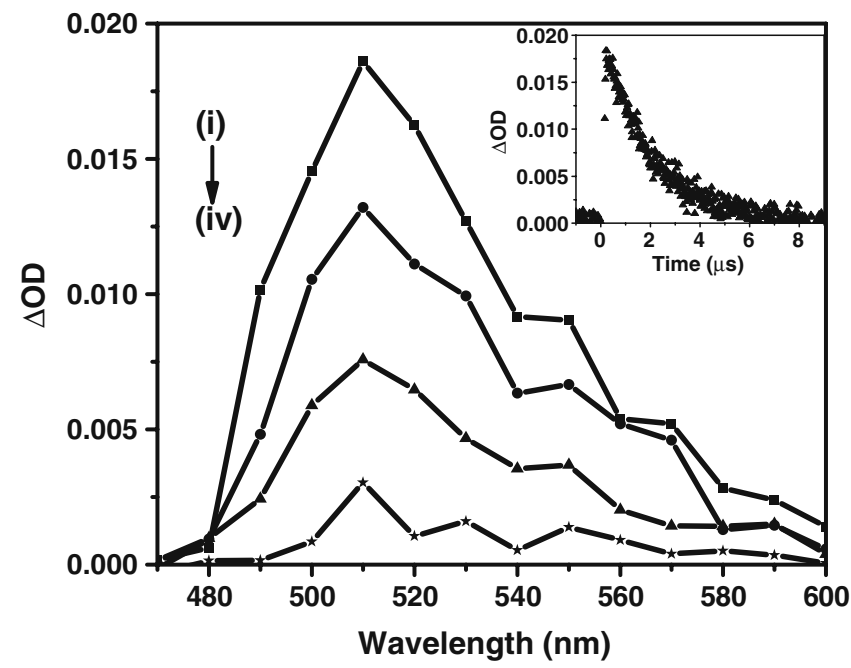

Figure 3. Transient absorption spectra of 9-phenylxanthenium tetrafluoroborate in acetonitrile recorded at $\mathrm{t}=0$ (i), $0 \cdot 65 \mu \mathrm{s}$ (ii), $1.7 \mu$ s (iii), $5 \cdot 2 \mu \mathrm{s}$ (iv) after $355 \mathrm{~nm}$ laser pulse excitation. The inset shows the decay profile at $510 \mathrm{~nm}$ for the triplet-triplet absorption.

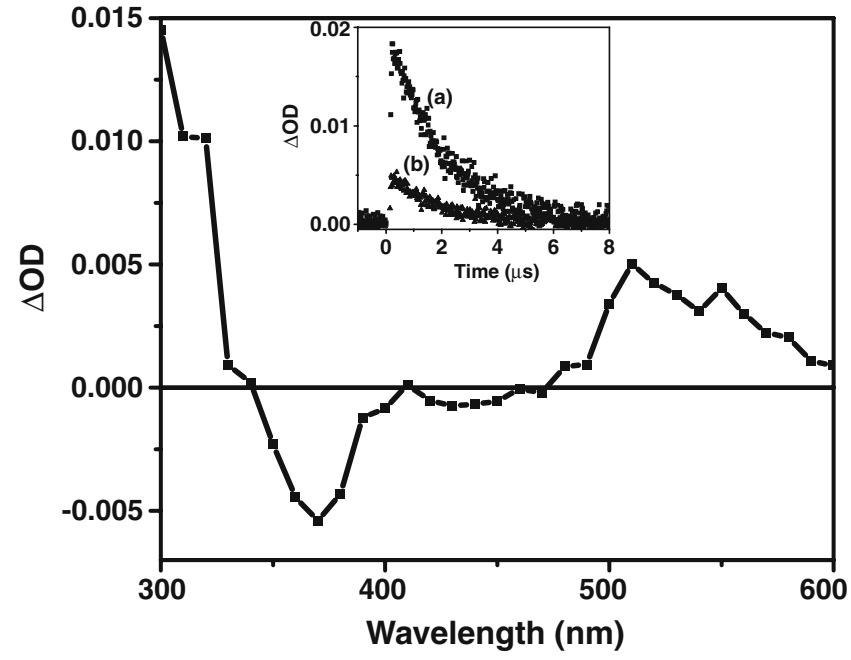

Figure 4. Transient absorption spectrum of 9phenylxanthenium tetrafluoroborate in acetonitrile $1 \mu$ s after $355 \mathrm{~nm}$ laser pulse excitation. The inset shows the comparison between the decay profiles at $300 \mathrm{~nm}$ (a) and $510 \mathrm{~nm}$ (b).

range of the transient absorption spectrum (figure 4) and by observing another band $\sim 300 \mathrm{~nm}$, which is previously characterized as a triplet-triplet absorption band of $\mathbf{P X}+{ }^{17,18}$ and whose decay behaviour is almost identical with that of the newly observed $510 \mathrm{~nm}$ band (inset of figure 4). The triplet nature of the transient is further confirmed by studying the effect of ethyl iodide (a heavy atom containing molecule) on the transient absorption band and on the fluorescence behaviour of the molecule. It is found that addition of ethyl iodide leads to significant reduction of the fluorescence lifetime of $\mathbf{P X}+$ and quenching of the lifetime of the transient species along with a decrease of the $\Delta O D$ value. The former is clearly a reflection of the enhancement of $S_{1}-T_{1}$ intersystem crossing rate and the latter is indicative of the enhancement of the decay of the $T_{1}$ state.

\subsection{Effect of oxygen}

Even though the fluorescence lifetime of $\mathbf{P X}+$ is quite long, it is quite insensitive to molecular oxygen. ${ }^{8,10}$ The oxygen quenching rate constant $\left(k_{q}<5 \times 10^{8} \mathrm{M}^{-1} \mathrm{~s}^{-1}\right)$ is much smaller than those observed for the singlet state of other hydrocarbons (typically of the order of $\left.10^{10} \mathrm{M}^{-1} \mathrm{~s}^{-1}\right){ }^{8}$ The transient species contributing to the new absorption $\sim 510 \mathrm{~nm}$, which we attribute to the triplet state of $\mathbf{P X}+$, is also reluctant to interact with oxygen (figure 5). On purging oxygen gas, the lifetime of the triplet state reduces from $2.1 \mu \mathrm{s}$ to $1.8 \mu \mathrm{s}$. The rate 
constant $\left(k_{\mathrm{T}}\right)$ of quenching of the triplet state by molecular oxygen, estimated by using the relation, $k_{\mathrm{T}}=$ $1 / \tau_{\mathrm{T}}\left[\mathrm{O}_{2}\right],{ }^{24}$ where $\tau_{\mathrm{T}}$ is the lifetime of the species undergoing triplet-triplet absorption equilibrated with air and $\left[\mathrm{O}_{2}\right]$ is the concentration of oxygen in solution, is found to be $1.8 \times 10^{8} \mathrm{M}^{-1} \mathrm{~s}^{-1}$ in acetonitrile (assuming $\left[\mathrm{O}_{2}\right] \sim 3 \mathrm{mM}$ under air saturation). ${ }^{25}$ This behaviour, i.e., oxygen quenching rate constant of $\mathbf{P X}+$ triplet to be smaller than the observed diffusion controlled values $\left(\sim 10^{10} \mathrm{M}^{-1} \mathrm{~s}^{-1}\right)$, is in accordance with the literature. ${ }^{17}$ Even though the triplet state energy of molecular oxygen is much lower than that of $\mathbf{P X}+(48 \mathrm{kcal} / \mathrm{mol}),{ }^{17}$ the energy transfer between the two is not favoured as the process requires formation of a charge transfer complex in which the electron deficient species, $\mathbf{P X}+$ has to serve as an electron donor. ${ }^{8}$

\subsection{Triplet-triplet energy transfer}

One of the methods of characterization of the triplet state of a species and determination of the triplet state parameters such as the extinction coefficient $\left(\varepsilon_{\mathrm{T}}\right)$ and quantum yield $\left(\phi_{\mathrm{T}}\right)$ is by triplet-triplet energy transfer process, in which the energy is transferred from the excited triplet state of the donor molecule to the triplet state of the acceptor. In order to characterize the newly observed band, we attempted sensitization of the triplet state of PX+ using several donor molecules with high triplet energies. Among the sensitizers chosen, benzo-

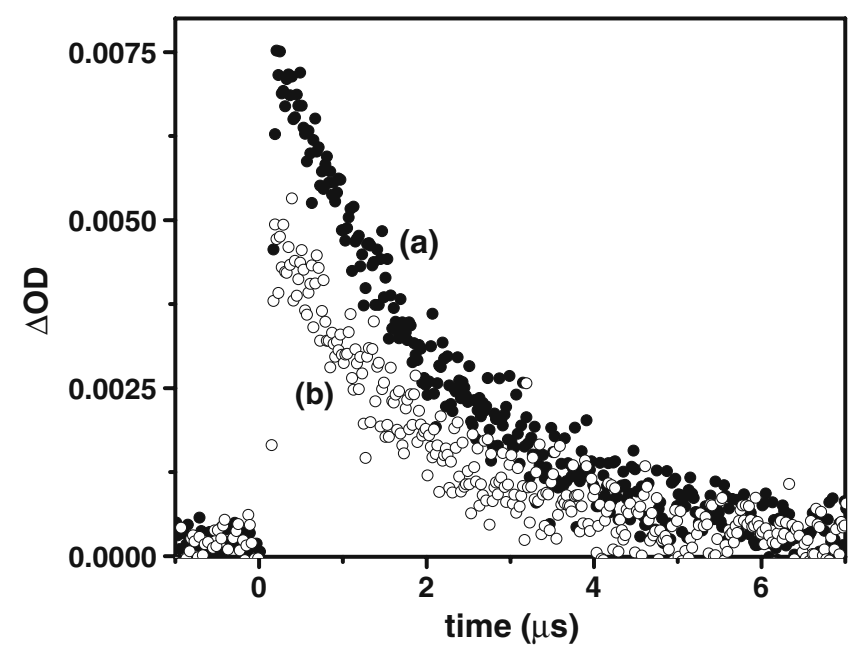

Figure 5. Triplet-triplet absorption decay profiles at $520 \mathrm{~nm}$ of (a) deoxygenated by purging argon gas and $\left(\tau_{\mathrm{T}}=2.05 \mu \mathrm{s}\right)$, (b) oxygen saturated solution $\left(\tau_{\mathrm{T}}=1 \cdot 81 \mu \mathrm{s}\right)$ of 9 phenylxanthenium tetrafluoroborate in acetinitrile. phenone and thioxanthone were expected to be quite appropriate for their high triplet energies, ${ }^{26-28}$ but we encountered problems due to trace quantity of moisture-induced generation of PX (scheme 1) during the addition of the sensitizer presumably because we avoided using any acid and preferred working with an acetonitrile solution of the 9-phenylxanthenium tetrafluoroborate salt. As, a solution of $\mathbf{P X}$ in $25 \% \mathrm{H}_{2} \mathrm{SO}_{4}$ consists entirely of the cation, ${ }^{16}$ we attempted sensitization of $\mathbf{P X}+$ triplet in acidic environment as well. However, in acidic environment, as commonly used high-energy sensitizers like benzophenone or thioxanthone, formed ketyl radicals from their triplet state, triplet sensitization of $\mathbf{P X}+$ was no longer possible. To circumvent this problem, we tried the arenes as sensitizers as these are not so much affected in acidic environment. However, high energy arenes such as naphthalene cannot be excited at $355 \mathrm{~nm}$ (excitation wavelength) and for other low energy arenes even though it was possible to adjust the concentration of the species to ensure that the donor to absorb $80-90 \%$ light, we could not avoid a situation in which direct excitation of $\mathbf{P X}+$ was completely avoided. Secondly, these arenes are electron-rich compounds, which undergo facile electron transfer reaction with electron deficient $\mathbf{P X}+{ }^{9,10}$ Hence, this excited state reaction between arenes and $\mathbf{P X}+$ competes with the triplet-triplet energy transfer process. Thus sensitizers like phenanthrene, triphenylene, coronene, fluoranthene, which possess high triplet energy, did not serve as donor in the triplet-triplet energy transfer process. We are searching for a suitable sensitizer for the triplet of $\mathbf{P X}+$.

\section{Conclusion}

This laser flash photolysis study on 9-phenylxanthenium tetrafluoroborate in neutral medium reveals a new feature that is attributed to the somewhat elusive triplet state of PX+. The newly identified triplet-triplet absorption band of $\mathbf{P X}+$, which is insensitive to oxygen and appears in the visible region, is well-separated from the absorption bands of 9-phenylxanthenyl radical or PX triplet and hence, interference from these species is avoided. The finding is thus expected to be helpful in the exploration of the triplet state properties and a better understanding of the photochemistry of $\mathbf{P X}+$.

\section{Acknowledgements}

This work has been supported by the J. C. Bose Fellowship of the Department of Science and Tech- 
nology, Government of India. Thanks are due to the University Grants Commission for the Centre for Advanced Studies Fellowship to SB.

\section{References}

1. Azarani A, Berinstain A B, Johnston L J and Kazanis S 1991 J. Photochem. Photobiol. A57 175

2. Bartl J, Steenken S, Mayr H and McClelland R A 1990 J. Am. Chem. Soc. 1126918

3. Johnston L J and Kanigan T $1990 \mathrm{~J}$. Am. Chem. Soc. 112 1271

4. McClelland R A, Kanagasabapathy V M, Banait N S and Steenken S 1991 J. Am. Chem. Soc. 1131009

5. Mecklenburg S L and Hilinski E F $1989 \mathrm{~J}$. Am. Chem. Soc. 1115471

6. Wan P and Krogh E 1989 J. Am. Chem. Soc. 1114887

7. Boyd M K, Lai H Y and Yates K 1991 J. Am. Chem. Soc. 1137294

8. Minto R E and Das P K 1989 J. Am. Chem. Soc. 111 8858

9. Samanta A, Gopidas K R and Das P K 1990 Chem. Phys. Lett. 167165

10. Samanta A, Gopidas K R and Das P K 1993 J. Phys. Chem. 971583

11. Valentino M R and Boyd M K 1993 J. Org. Chem. 58 5826
12. Bedlek J M, Valentino M R and Boyd M K 1996 J. Photochem. Photobiol. A94 7

13. Samanta A, Gopidas K R and Das P K 1993 Chem. Phys. Lett. 204269

14. Feldman M R and Thame N G 1979 J. Org. Chem. 441863

15. Wan P, Yates K and Boyd M K 1985 J. Org. Chem. 50 2881

16. McClelland R A, Banait N and Steenken S 1989 J. Am. Chem. Soc. 1112929

17. Johnston L J and Wong D F 1992 Can. J. Chem. 70280

18. Johnston L J and Wong D F 1993 J. Phys. Chem. 97 1589

19. Dauben H and Harmon K 1960 J. Org. Chem. 251442

20. Samanta A and Paul A 2006 J. Phys. Chem. B111 1957

21. Samanta A and Bhattacharya B 2007 Chem. Phys. Lett. 442316

22. Berger R M and Weir D 1990 Chem. Phys. Lett. 169 213

23. Samanta A, Bhattacharyya K, Das P K, Kamat P V, Weir D and Hug G L 1989 J. Phys. Chem. 933651

24. Kruk N N and Nichiporovich I N 2004 J. Appl. Spec. 71 343

25. Murov S L 1973 Handbook of Photochemistry (New York: Marcel Dekker)

26. Kasha M and Lewis G N 1944 J. Am. Chem. Soc. 66 2100

27. Wilkinson F 1962 J. Phys. Chem. 662569

28. Lamola A A, Herkstroeter W G and Hammond G S 1964 J. Am. Chem. Soc. 864537 